\title{
1 Besonderheiten und Probleme bei der Anästhesie des adipösen Kindes
}

\author{
Claudia Philippi-Höhne
}

Etwa 15\% aller Kinder und Jugendlichen in Deutschland sind übergewichtig. $6 \%$ sind sogar adipös und die Tendenz ist steigend. Auch in der Kinderanästhesie wird diese Thematik daher zunehmend relevant: Aufgrund der Adipositas kann es während einer Allgemeinanästhesie zu Komplikationen kommen. Der folgende Beitrag fasst den Wissensstand auf diesem Gebiet zusammen. Es werden die Definition für Adipositas im Kindesalter gegeben und Ursachen erläutert, über Adipositas-assoziierte Begleiterkrankungen informiert und das entsprechende anästhesiologische Management dargestellt.

\subsection{Das übergewichtige Kind}

Bei Kindern verwendet man zur Einteilung von Übergewicht und Adipositas wie bei Erwachsenen den Body-Mass-Index (BMI). Es ist jedoch abhängig vom Alter, dem Geschlecht und der ethnischen Herkunft, ob ein Kind als normal- oder übergewichtig einzustufen ist. Durch die körperliche Entwicklung verändert sich das Verhältnis von Körpermasse zu Körpergewicht: Ein 3-jähriges Mädchen mit einem BMI von 19,4 würde unter Adipositas leiden, während ein 11-jähriges Mädchen mit diesem BMI normalgewichtig wäre. Anhand dieses Beispiels wird deutlich, dass die Grenzen der Erwachsenen auf Kinder und Jugendliche nicht übertragbar sind. Sogenannte Perzentilen berücksichtigen diese Faktoren. Es handelt sich hierbei um Kurven, die Auskunft über die pro- zentuale Verteilung des BMI geben, jeweils auf ein Geschlecht und ein Land bezogen. In Deutschland wurde die 9o. Perzentile als Grenze zum Übergewicht definiert, die 97. Perzentile als Grenze zur Adipositas. Ab der 99,5. Perzentile spricht man von Adipositas per magna (Kromeyer-Hausschild 2001; Cole et al. 2000).

Die Perzentilen sind jedoch lediglich statistische Ermittlungen, welche die Verteilung der BMI-Werte innerhalb einer Bevölkerungsgruppe zu einem bestimmten Zeitpunkt beschreiben: Die go. Perzentile besagt, dass 90\% aller Kinder einen niedrigeren BMI haben. Die 50. Perzentile repräsentiert den Mittelwert. Hier wird deutlich, dass die Kurven sich somit im Laufe der Zeit verschieben können: Steigt die Anzahl der übergewichtigen Kinder an, so verändert sich die Grenzperzentile zum Übergewicht.

\section{2 Ätiologie der Adipositas bei Kindern}

Für die Entstehung von Übergewicht im Kindes- und Jugendalter gibt es verschiedene Ursachen (u.a Kiess et al. 2001; Coon et al. 2001).

Sozio-kulturelle Ursachen

- wenig Bewegung durch Beschäftigung vor Computer und Fernseher

- übermäßiges Nahrungsangebot: Fast Food, Süßigkeiten

- Einfluss von Werbung 
Essen zur Problembewältigung bei psychologischen Problemen

- binge eating disorder (periodische Fressanfälle)

- Bulimia nervosa

\section{Medizinische Ursachen}

- Genetische Defekte/Syndrome

- Polymorphismen und Mutationen z.B. von: Leptin, Thyreotropin Releasing Hormone (TRH), Proopiomelanocortin (POMC) u.a.

- Prader-Labhart-Willi-Syndrom

- Bardet-Biedl-Syndrom

- Fröhlich-Syndrom

\section{Stoffwechselstörungen \\ - Cushing-Syndrom \\ - Hypothyreose \\ - Primärer Hyperinsulinismus \\ - Pseudohyperparathyreoidismus}

\section{Pharmaka}

- Glukokortikoide

- Insulin

\author{
Hypothalamischer Symptomkomplex (Hirnschäden) \\ - Tumoren \\ - Entzündungen \\ - Traumata
}

\section{Erkrankungen mit Bewegungsmangel \\ - Muskeldystrophien im Endstadium (Typ Duchenne) \\ - Orthopädische Fehlbildungen}

Bei bis zu 90\% der Kinder führen vor allem körperliche Inaktivität und eine falsche Ernährung zu einer positiven Energiebilanz und damit zu Übergewicht und Adipositas. Das Essverhalten von Kindern unterliegt vielen Einflüssen. Die Umwelt und die Erziehung spielen eine große Rolle, aber auch die Psyche beeinflusst Art und Menge der Nahrung (Delmas et al. 2007; Hölling u. Schlack 2007; Weber et al. 2008). In vielen Fällen ist Übergewicht innerhalb einer Familie nicht auf genetische Ursachen, sondern vielmehr auf das gleiche Essverhalten von Eltern und Kindern zurückzuführen.

In etwa $5 \%$ aller Fälle hat Übergewicht bei Kindern eine medizinische Störung als Ursache (u.a. Kiess et al. 2001; Veyckemans 2008). Des Weiteren werden Risikofaktoren diskutiert, bei denen der genaue Mechanismus noch nicht bekannt ist. Man nimmt jedoch an, dass ein hohes Geburtsgewicht (Gillman et al. 2003), wenig Schlaf, der Einfluss von Nikotin während der Schwangerschaft (Toschke et al. 2003) und Flaschenernährung im Säuglingsalter (Gillman et al. 2001) Stoffwechselvorgänge beeinflussen und hohe Insulinspiegel hervorrufen. Das wiederum kann im Laufe der Entwicklung in einem erhöhten Gewicht resultieren.

\subsection{Prävalenz}

Daten aus den USA (2007-2008) zeigen, dass etwa 17\% der Kinder und Jugendlichen im Alter von 2-18 Jahren adipös sind. Unter Vorschulkindern ist die Prävalenz von $5 \%$ (1976-80) auf 10,4\% (2007-8) gestiegen. Im gleichen Zeitraum stieg die Prävalenz unter Schulkindern von $6,5 \%$ auf $19,6 \%$ und unter Jugendlichen von 5\% auf 18,1\% (http://www.cdc.gov/obesity/ childhood/index.html).

In Deutschland kam die letzte große bundesweite prospektive Studie des Robert Koch-Institutes (2006) zu dem Ergebnis, dass $15 \%$ der 3- bis 17-jährigen in Deutschland übergewichtig sind. 6,3\% dieser Altersgruppe leiden unter Adipositas. Daten aus dem Jahr 2009 zeigen, dass nunmehr 17\% der Kinder und Jugendlichen übergewichtig und $7,7 \%$ adipös sind. Betroffen sind besonders Jugendliche im Alter zwischen 14 und 17 Jahren. Zwischen den alten und neuen Bundesländern sowie zwischen den Geschlechtern gibt es hingegen keine signifikanten Unterschiede (Kurth et al. 2007).

\subsection{Pathophysiologische Veränderungen}

Wie auch bei Erwachsenen führt Adipositas bei Kindern und Jugendlichen zu Veränderungen an verschiedenen Organsystemen. Es ist jedoch nicht bekannt, wie lange und in welchem Ausmaß die Adipositas bestehen muss, um Organmanifestation nach sich zu ziehen. Tabelle 1 gibt einen Überblick über die anästhesierelevante Pathophysiologie.

\subsection{Präoperative Diagnostik und Prämedikation}

Die präoperative Diagnostik orientiert sich wie bei normalgewichtigen Kindern an dem geplanten Eingriff und der Anamnese. Es sollte ausdrücklich nach der körperlichen Belastbarkeit sowie nach Symptomen der Schlafapnoe gefragt werden. Hierzu zählen: - Schnarchen,

- ein unruhiger Schlaf mit häufigem nächtlichen Erwachen und

- ein daraus resultierender gestörter Tag-NachtRhythmus mit verstärkter Müdigkeit am Tag. 
Tab. 1 Anästhesierelevante pathophysiologische Veränderungen verschiedener Organsysteme

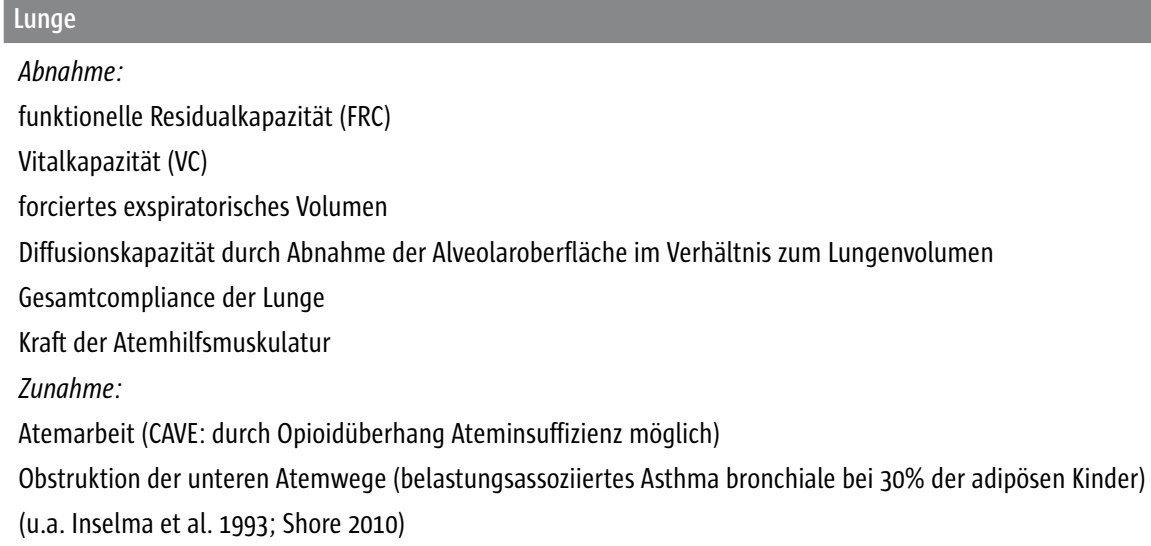

Zunahme der Wanddicke der Intima, der Steifheit der A. carotis und kompensatorische Erweiterung des Gefäßlumens bei frühzeitiger Arteriosklerose bereits im Schulkindalter (lanuzzi et al. 2004 und 2008)

Steigerung der Sympathikusaktivität

häufiger Blutdruckanstieg therapiebedürftige Hypertonie (Sorof u. Daniels 2002)

Zunahme der Ruheherzfrequenz

Verstärkung der Vaskularisierung des Fettgewebes

Anstieg des Herzminutenvolumens und des Blutvolumens

Entwicklung einer linksventrikulären Hypertrophie (Hanevold et al. 2004) und Dysfunktion (Harada et al. 2001)

Entstehung einer rechtsventrikulären Insuffizienz aufgrund eines pulmonalen Hypertonus

\section{Gastrointestinaltrakt}

widersprüchliche Daten zur Inzidenz des gastro-ösophagealen Refluxes, erhöhte Rate (Tait et al. 2008), kein Unterschied zwischen Normalgewichtigen und adipösen Kindern (Elitsur et al. 2009)

Diabetes mellitus Typ II und metabolisches Syndrom bei 50\% der stark übergewichtigen Jugendlichen (Weiss et al. 2004)

Fettleber, selten Leberfunktionsstörung und erhöhte Alanin-Aminotransferase, aber reversible hepatische Steatose bis Fibrose möglich (Sagi et al. 2007)

\section{Sonstiges}

Pickwick-Syndrom: Adipositas-assoziiertes Hypoventilationssyndrom

chronische Hypoxämie und Hyperkapnie mit tagesbetonter Müdigkeit, Polyzythämie und pulmonalem Hypertonus

Schlafapnoesyndrom: bis zu 17\% bei einem BMI > 150. Perzentile (Veyckemans 2008)

Eine Echokardiografie sollte bei Verdacht auf kardiale Erkrankungen bzw. eine pulmonale Hypertonie angeordnet werden. Besteht der Verdacht auf Herzrhythmusstörungen, empfiehlt es sich, ein EKG zu schreiben. Gibt es Anhaltspunkte für pulmonale Funktionseinschränkungen und ist eine umfangreiche Operation geplant, so kann eine Lungenfunktionsdiagnostik Hinweise auf eine potenzielle Optimierung der Medikation geben. Die Labordiagnostik orientiert sich an den allgemein gültigen Empfehlungen für die Kinderanästhesie (Becke et al. 2007).

Zur Anxiolyse ist bei Kindern die Gabe von Midazolam, einem Benzodiazepin, am gebräuchlichsten. Eine potenzielle Gefahr besteht jedoch bei bekanntem Schlafapnoesyndrom. Es kann durch Reduktion des Tonus der Zungengrundmuskulatur zur Verlegung der Atemwege mit der Folge einer Apnoe kommen. Sollte aufgrund der Anamnese dieses Risiko be- 
stehen, ist es wichtig, die Indikation der Medikamentengabe sehr streng zu stellen und wenn, dann nur unter Überwachungsmaßnahmen (v.a. Pulsoxymetrie) und der Möglichkeit der Sauerstoffapplikation/Beatmung durchzuführen. Anderenfalls sollte darauf verzichtet werden.

\subsection{Anästhesiologisches Management}

\section{Monitoring}

Beim überwiegenden Anteil der Eingriffe ist das Basismonitoring ausreichend:

- Dreikanal-EKG zur Überwachung von Herzrhythmus und -frequenz

- Nicht-invasive Blutdruckmessung mittels einer Blutdruckmanschette

- Pulsoxymetrie zur Überwachung der Sauerstoffsättigung

- Kapnometrie zur Messung der $\mathrm{CO}_{2}$-Konzentration

- Temperatur

Bei der nicht-invasiven Blutdruckmessung sollte auf eine korrekte Manschettengröße geachtet werden, die sich am Umfang der jeweiligen Extremität orientieren sollte. Bei Normalgewichtigen wird empfohlen, sich an der Länge z.B. des Oberarmes zu orientieren, bei adipösen Armen würde man so zu ungenauen Werten gelangen. Die Kammerbreite der Manschette sollte idealerweise $40 \%$ des Umfangs der zu messenden Extremität betragen (Sorof $u$. Daniels 2002). Zu kleine Manschetten führen zu falsch hohen Werten, zu große Manschetten zu falsch tiefen Werten.

Die Bezeichnungen auf den Manschetten sind nicht in jedem Fall verwendbar: Adipöse Kinder benötigen ggf. größere Manschetten als ihre Altersgenossen.

- Ist keine passende Manschette für den Oberarm vorhanden, sollte man an einer anderen Stelle messen (z.B. Oberschenkel).

Eine invasive Blutdruckmessung ist nur selten erforderlich und wird vor allem durch den Umfang des chirurgischen Eingriffes bestimmt. Bei schwerwiegenden kardialen Vorerkrankungen des Patienten sollte sie großzügig eingesetzt werden. So können selbst kleinere Blutdruckschwankungen zeitnah und korrekt registriert werden und die Oxygenierung mittels arterieller Blutgasanalyse bestimmt werden.
Neben dem Standardmonitoring kann bei adipösen Kindern ein Neuromonitoring zur Überwachung der Narkosetiefe hilfreich sein, um die adäquate Dosierung der Hypnotika zu applizieren, denn die gebräuchlichen Hypnotika interagieren unterschiedlich stark mit dem Fettgewebe (s.u.).

\section{Intravenöser Zugang}

Ein intravenöser Zugang gehört zu jeder Anästhesie. Bei adipösen Patienten kann die Punktion durch starkes Unterhautfettgewebe erschwert sein und häufig sehr viel Zeit in Anspruch nehmen. In einer prospektiven Studie konnte gezeigt werden (Nafiu et al. 2010), dass die Anlage des intravenösen Zugangs bei adipösen Kindern beim ersten Versuch häufig misslang und erst erfolgreich war, wenn dann die Vene an der Innenseite des Handgelenks punktiert wurde. Das Berücksichtigen dieses Wissens hat zwei Vorteile: zum einen kann an dieser Stelle durch Auftragen einer Lokalanästhetika-haltigen Salbe der Punktionsschmerz deutlich reduziert werden, zum anderen können frustrane Punktionsversuche dem Patienten Stress und dem Anästhesieteam Zeit ersparen.

Bei großen Operationen ist ein zentraler Venenkatheter (ZVK) indiziert, sofern er postoperativ für Medikamentengabe (Antibiotika, Analgetika u.a.) oder Ernährung benötigt wird. Auch hier kann die Anlage durch die Adipositas schwierig sein. Die Punktion sollte darum unter Ultraschallkontrolle erfolgen.

\section{Bei massiv adipösen Kindern ist die Anlage eines ve- nösen Zugangs häufig handgelenknah an der Innen- seite des Unterarms erfolgreich.}

\section{Hypnotika}

Sowohl bei Erwachsenen als auch bei Kindern und Jugendlichen ist es anzustreben, Hypnotika zu verwenden, die gut steuerbar sind und eine kurze Aufwachzeit ermöglichen.

Desfluran scheint von den heute verwendeten inhalativen Anästhetika am besten steuerbar zu sein. Sowohl bei Desfluran als auch bei Sevofluran wurden in Kombination mit einer Überwachung der Narkosetiefe mittels Neuromonitoring ähnliche Aufwachzeiten wiebei Erwachsenen beobachtet (Arain et al. 2005). Das adipöse Kind stellt jedoch eine Besonderheit dar, da es zu Bronchoobstruktionen neigt. Desfluran 
kann den Atemwegswiderstand bei Kindern mit hyperreagiblem Atemwegssystem erhöhen und sein Einsatz ist daher wenig sinnvoll. Sevofluran hingegen wirkt zum Teil bronchodilatatorisch und scheint diesbezüglich vorteilhaft zu sein (von Ungern-Sternberg et al. 2008).

Prinzipiell ist auch die Verwendung von Propofol möglich. Die Dosierung erfolgt anhand des Gesamtgewichts.

\section{Opioide, Muskelrelaxantien}

Opioide sollten bei adipösen Kindern mit besonderer Vorsicht eingesetzt werden, um einen postoperativen Opioidüberhang zu vermeiden. Sufentanil hat bei kontinuierlicher bzw. repetitiver Gabe eine verlängerte Halbwertszeit. Die Dosis sollte daher entsprechend angepasst werden (Schwartz et al. 1991). Auch die Dosis von Alfentanil sollte bei adipösen Patienten verringert werden. Fentanyl hingegen weist bei Adipositas eine nahezu unveränderte Pharmakokinetik auf. Remifentanil zeichnet sich durch eine schnelle Elimination aus - auch bei Adipositas bleibt diese Eigenschaft relativ unverändert erhalten. Die Dosierung von Remifentanil richtet sich nach dem Idealgewicht. Genaue Daten zur Empfehlung für die Dosierung der Hypnotika und Opioide bei Übergewichtigen gibt es bislang nur für Erwachsene.

Auch für die Cruppe der Muskelrelaxantien liegen bisher nur Ergebnisse für Erwachsene vor: Die Untersuchungen an adipösen Patienten kamen zu dem Ergebnis, nicht-depolarisierende Muskelrelaxantien (z.B. Vecuronium, Rocuronium und Cisatracurium) anhand des Idealgewichts zu dosieren. Anderenfalls ist mit einer unerwünscht langen Wirkdauer zu rechnen.

\section{Ambulante Versorgung}

Für ambulante Eingriffe bei adipösen Kindern gelten die gleichen Bedingungen wie bei adipösen Erwachsenen: Sind keine weiteren Begleiterkrankungen bekannt, ist eine ambulante Versorgung gut möglich. Sind jedoch Adipositas-assoziierte Komorbiditäten vorhanden, ist eine stationäre Betreuung anzustreben, da mit intra- oder postoperativen Komplikationen zu rechnen ist (Strauß u. Höhne 2008). Studien liefern bislang keine belastbaren Zahlen, ab welchem Grad der Adipositas von ambulanten Eingriffen abzuraten ist.

\section{Sedierung}

Eine Sedierung bei einem adipösen Kind ist eine Herausforderung für den durchführenden Anästhesisten oder Intensivmediziner!

Werden die Hypnotika anhand des Gesamtkörpergewichts dosiert, so führt dies häufig zu Überdosierung. Orientiert man sich am Körpergewicht ohne Fett (lean body weight), kommt es häufig zu Unterdosierung und unzureichenden Untersuchungsbedingungen (Baker u. Yagiela 2006). Bei diesen Patienten sollte ein besonderes Augenmerk auf die $\mathrm{Me}$ dikamentenauswahl (hydrophil und kurze Halbwertszeit), die langsame Titrierung der Medikamente und auf die korrekte Lagerung des Patienten gelegt werden. Damit kann die Häufigkeit von Komplikationen vermindert werden.

Für eine Sedierung eignet sich Propofol, ist eine Analgosedierung notwendig kann Remifentanil verwendet werden. In jedem Fall muss mit Hypoventilation, Atemwegsverlegung und Apnoe gerechnet werden.

\subsection{Perioperative Komplikationen}

Bei adipösen Patienten werden während Allgemeinnarkosen vermehrt Probleme und Komplikationen beobachtet. Für Erwachsene ist dies bereits in etlichen Untersuchungen erwiesen. Klinische Studien für übergewichtige und adipöse Kinder hingegen liegen bislang nur wenige vor (Nafiu et al. 2007, 2009; Setzer u. Saade 2007; Tait et al. 2008). Hauptsächlich tragen die Adipositas-assoziierten Nebenerkrankungen, aber auch das Fettgewebe und seine Verteilung unmittelbar zur erhöhten Inzidenz von Komplikationen bei, auf die im Folgenden detailliert eingegangen werden soll.

\section{Atemweg}

Bei übergewichtigen und adipösen Kindern treten Atemwegsobstruktionen häufiger auf als bei Normalgewichtigen. Eine prospektive Analyse von Tait et al. (2008) wies in diesem Zusammenhang den häufigeren Einsatz von Guedel- und Wendl-Tuben bei adipösen Kindern nach. Auch der Esmarch-Handgriff zur Unterstützung der Atmung bei Verlegung der oberen Atemwege wurde in der adipösen Cruppe häufiger angewandt.

Laryngospasmen wurden bei übergewichtigen Kindern in einer retrospektiven Studie ebenfalls häufi- 
Tab. 2 Übersicht der vier relevanten Studien über perioperative Atemwegskomplikationen bei adipösen Kindern

\begin{tabular}{|c|c|c|c|c|}
\hline & Tait et al. 2008 & Setzer u. Saade 2007 & Nafiu et al. 2007 & Nafiu et al. 2009 \\
\hline & $n=2025$ & $n=1133$ & $n=6094$ & $n=2170$ \\
\hline & prospektiv & retrospektiv & retrospektiv & retrospektiv \\
\hline Art der Eingriffe & $\begin{array}{l}\text { alle außer } \\
\text { kardiochirurgisch }\end{array}$ & Zahnmedizin & Kinderchirugie & HNO \\
\hline \multirow{2}{*}{$\begin{array}{l}\text { Prävalenz von } \\
\text { Übergewicht } \\
\text { und Adipositas }\end{array}$} & Übergewicht 17,3\% & Adipositas 8,8\% & Übergewicht 17,2\% & Übergewicht 13,1\% \\
\hline & Adipositas 14,5\% & & Adipositas 14,4\% & Adipositas 7,5\% \\
\hline \multirow[t]{8}{*}{$\begin{array}{l}\text { erhöhte Inzidenz } \\
\text { von Komplikationen }\end{array}$} & $\begin{array}{l}\text { nur bei adipösen Kindern: } \\
\text { schwierige }\end{array}$ & $\begin{array}{l}\text { Sättigungsabfall } \\
0,2 \text { vs. } 2 \%\end{array}$ & \multirow{8}{*}{$\begin{array}{l}\text { schwierige } \\
\text { Maskenventilation } \\
3,6 \text { vs. 7,4\% } \\
\text { schwierige } \\
\text { Laryngoskopie } \\
0,2 \text { vs. 1,3\% } \\
\text { längerer Aufenthalt } \\
\text { im Aufwachraum }\end{array}$} & $\begin{array}{l}\text { schwierige Masken- } \\
\text { ventilation } 15,2 \text { vs. } 31,6 \%\end{array}$ \\
\hline & Maskenventilation & ungeplante & & schwierige Intubation \\
\hline & 2,1 vs. $8,7 \%$ & stationäre Aufnahme & & 11,9 vs. $27,4 \%$ \\
\hline & Husten 4,3 vs. $7,1 \%$ & & & Atemwegsobstruktion \\
\hline & Atemwegsobstruktion & & & 0,2 vs. $5,9 \%$ \\
\hline & 11,2 vs. $18,9 \%$ & & & Laryngospasmus 0,2 vs. $2,1 \%$ \\
\hline & Sättigungsabfall & & & Sättigungsabfall \\
\hline & 9,1 vs. $16,8 \%$ & & & 30,9 vs. $40,4 \%$ \\
\hline
\end{tabular}

ger beobachtet als bei Normalgewichtigen (Nafiu et al. 2009), wobei dies in dem speziellen Kollektiv der HNO-Patienten gezeigt wurde. In anderen chirurgischen Bereichen ist dies nicht nachweisbar.

Tabelle 2 zeigt die vier bisher vorliegenden Studien aus den USA, die eine erhöhte Inzidenz an Atemwegskomplikationen beobachten, wobei die prospektive Studie von Tait et al. (2008) am aussagekräftigsten ist.

\section{Maskenbeatmung und Intubation}

Die erhöhte Inzidenz der schwierigen Maskenventilation, der Atemwegsverlegung und der schwierigen Larnygoskopie bei adipösen Kindern kann folgende Ursachen haben:

- MRT-Aufnahmen zeigen eine Verminderung des pharyngealen Raumes durch die Einlagerung von Fettgewebe. Verschiedene Strukturen sind betroffen, wie die Uvula, die Tonsillen, die Zunge, der aryepiglottische Raum und die seitlichen pharyngealen Wände (Benumof 2004).

- Adipöse Kinder mit Schlafapnoe haben ein höheres Tonsillenvolumen und ein höheres Tonsillengewicht als Normalgewichtige (Wang et al. 2010). Das kann eine Erklärung für die höhere Inzidenz der Atemwegsobstruktion sein, denn alle Hypnotika bewirken ein Kollabieren der pharyngealen Strukturen.
- Bei stark adipösen Kindern kann potenziell das oberflächliche Fett an den Halsweichteilen aufgrund der externen Kompression das Offenhalten des Pharynx einschränken. Bei Erwachsenen konnte gezeigt werden, dass die Erhöhung des extraluminalen Druckes durch das Fett zur Kompression der oberen Luftwege führt (Benumof 2004). Dieser Effekt kommt nach Applikation von Hypnotika verstärkt zum Tragen.

- Die Fettablagerungen im Nacken können die Reklination einschränken und die vergrößerte Zunge kann die Larnygoskopie und Intubation erschweren. Dies konnte zumindest in den retrospektiven Studien von Nafiu et al. (2007; 2009) gezeigt werden.

In drei von vier Untersuchungen weisen adipöse Kinder vermehrt Sättigungsabfälle vor allem während der Ein- und Ausleitung, aber auch intraoperativ auf. Dies entspricht auch den eigenen klinischen Erfahrungen. Begründet ist dies in veränderten Lungenvolumina, insbesondere einer stark eingeschränkten funktionellen Residualkapazität (FRC) (Inselma et al. 1993; Tait et al. 2008). Hinzu kommt eine verstärkte Neigung zur Bildung von Atelektasen. 
- Für eine optimale Ventilation ist daher ein ausrejchender PEEP erforderlich.

- Eine gute Präoxygenierung ist ebenfalls wichtigwenn es toleriert wird, kann auch hier ein PEEP eingestellt werden.

- Die Oberkörperhochlagerung zur Ein- und Auslejtung und im Aufwachraum ist zu empfehlen. Die Abnahme der FRC durch den intraabdominellen Druck ist in dieser Position geringer und starke Hypoxien können potenziell vermieden werden.

Übergewichtige Kinder sind insbesondere bei Narkoseein- und -ausleitungen durch eine Hypoxämie gefährdet.

\section{Herz-Kreislauf-System}

Perioperative kardiovaskuläre Komplikationen werden sehr selten beobachtet. Zwei aktuelle Studien weisen jedoch auf folgende Aspekte hin: eine höhere Inzidenz von Hypotension nach Narkoseinleitung und ein schlechteres Outcome nach kardiopulmonaler Reanimation.

Nafiu et al. (2010a) beobachteten bei 19.400 Kindern (2-17 Jahre) eine erhöhte Inzidenz von Hypotension vor chirurgischem Stimulus bei adipösen Kindern und Jugendlichen von 40,9\% im Vergleich zu Normalgewichtigen mit 31,4\%. Eine mögliche Erklärung könnte eine linksventrikuläre Dysfunktion sein, die präoperativ asymptomatisch ist (Harada et al. 2001).

Die Daten müssen jedoch kritisch hinterfragt werden. So wurden die Daten aus elektronisch im klinischen Alltag erhobenen Protokollen entnommen, die Anästhesietechnik wurde nicht standardisiert, die korrekte Größe der Blutdruckmanschette war nicht kontrolliert und die präoperative Flüssigkeitszufuhr und der Volumenstatus wurden nicht berücksichtigt.

Srinivasan et al. (2010) analysierten 1.477 kardiopulmonale Reanimationen bei Kindern und Jugendlichen bis zum Alter von 18 Jahren. Die Prävalenz der Adipositas lag in diesem Kollektiv bei $17 \%$ und war verbunden mit einer niedrigeren Überlebensrate zum Zeitpunkt der Entlassung aus dem Krankenhaus nach Reanimation in der Klinik. Potenzielle Erklärungen seitens der Autoren sind:

- Die Effektivität der Herzdruckmassage in Bezug auf Kraft und Eindrücktiefe könnte aufgrund der Adipositas eingeschränkt sein.
- Die Dosierung der Medikamente anhand des totalen Körpergewichts hat sich negativ ausgewirkt.

- Die Defibrillation ist gewichtsadaptiert (2 J/kg), sodass zu hohe Energien eingesetzt wurden.

\section{Gastrointestinaltrakt}

Gastrointestinale Probleme und Komplikationen sind im Kindes- und Jugendalter ebenfalls sehr selten. In der Studie von Tait et al. (2008) wurde die Diagnose gastro-ösophagealer Reflux bei den adipösen Kindern häufiger beschrieben. Eine aktuelle weitere Analyse bei Kindern (Elitsur et al. 2009) kann das allerdings nicht bestätigen. Auch die Aspirationsrate ist nicht erhöht (Borland et al. 1998). Zudem zeigt sich, dass das Mageninhaltsrestvolumen nach $6 \mathrm{~h}$ Nüchternheit von Nahrung und 2 h Nüchternheit von klarer Flüssigkeit sich nicht zwischen normalgewichtigen und adipösen Kindern unterscheidet (Cook-Sather et al. 2009). Wie oben beschrieben, ist die Hypoxiegefahr schon während der normalen Einleitung erhöht. Eine rapid-sequence-induction (RSI) wird bei nüchternen adipösen Kindern demzufolge nicht empfohlen.

\section{Wegen der ohnehin erhöhten Gefahr der Hypoxämie bei Narkoseeinleitung ist eine „Ileuseinleitung" bei nüchternen adipösen Kindern nicht empfehlenswert.}

\section{Fazit}

Im anästhesiologischen Alltag werden zunehmend Kinder mit Übergewicht und Adipositas versorgt. Diese haben aufgrund der pathophysiologischen Veränderungen ein erhöhtes perioperatives Risiko, unter anderem Atemwegskomplikationen zu erleiden. Macht man sich diese Tatsache bewusst, sind die Kinder nicht als gesund einzustufen und mit erhöhter Aufmerksamkeit zu betreuen.

\section{Literatur}

Arain SR, Barth CD, Shankar H, Ebert TI (2005) Choice of volatile anesthetic for the morbidly obese patient: sevoflurane or desflurane. I Clin Anesth 17, 413-419

Baker S, Yagiela JA (2006) Obesity: a complicating factor for sedation in children. Pediatr Dent 28 487-493

Becke K, Giest J, Strauß JM (2007) Handlungsempfehlungen zur präoperativen Diagnostik, Impfabstand und Nüchternheit im Kindesalter. Anästh Intensivmed 48, S. 62-66 
Benumof JL (2004) Obesity, sleep apnea, the airway and anesthesia. Curr Opin Anaesthesiol 17, 21-30

Borland LM, Sereika SM, Woelfel SK, Saitz EW, Carrillo PA, Lupin JL, Motoyama EK (1998) Pulmonary aspiration in pediatric patients during general anesthesia: incidence and outcome. I Clin Anesth 10, 95-102

Cole T), Bellizzi MC, Flegal KM, Dietz WH (2000) Establishing a standard definition for child overweight and obesity worldwide: international survey. BMI 320, 1-6

Cook-Sather SD, Gallagher PR, Kruge LE, Beus JM, Ciampa BP, Welch KC, Shah-Hosseini S, Choi JS, Pachikara R, Minger K, Litman RS, Schreiner MS (2009) Overweight/obesity and gastric fluid characteristics in pediatric day surgery: implications for fasting guidelines and pulmonary aspiration risk. Anesth Analg 109, 727-736

Coon KA, Goldberg I, Rogers BL, Tucker KL (2001) Relationships between use of television during meals and children's food consumption patterns. Pediatrics 107 , e7

Delmas C, Platat C, Schweitzer B, Wagner A, Oujaa M, Simon C (2007) Association between television in bedroom and adiposity throughout adolescence. Obesity 15, 2495-2503

Elitsur Y, Dementieva Y, Elitsur R, Rewalt M (2009) Obesity is not a risk factor in children with reflux esophagitis: a retrospective analysis of 738 children. Metab Syndr Relat Disord 7, 211-214

Gillman MW, Rifas-Shiman S, Berkey CS, Field AE, Colditz GA (2003) Maternal gestational diabetes, birth weight, and adolescent obesity. Pediatrics 111, e221-226

Gillman MW, Rifas-Shiman SL, Camargo CA), Berkey CS, Frazier AL, Rockett HR, Field AE, Colditz GA (2001) Risk of overweight among adolescents who were breastfed as infants. JAMA 285, 2461-2467

Hanevold C, Waller I, Daniels S, Portman R, Sorof J, International Pediatric Hypertension Association (2004) The effects of obesity, gender, and ethnic group on left ventricular hypertrophy and geometry in hypertensive children: a collaborative study of the International Pediatric Hypertension Association. Pediatrics $113,328-333$

Harada K, Orino T, Takada G (2001) Body mass index can predict left ventricular diastolic filling in asymptomatic obese children. Pediatr Cardiol 22, 273-278

Hölling H, Schlack R (2007) Essstörungen im Kindes- und Jugendalter. Erste Ergebnisse aus dem Kinder- und Jugendgesundheitssurvey (KiGGS). Bundesgesundheitsbl 50, 794-799

lannuzzi A, Licenziati MR, Acampora C, De Michele M, lannuzzo G, Chiariello G, Covetti G, Bresciani A, Romano L, Panico S, Rubba $P$ (2008) Carotid artery wall hypertrophy in children with metabolic syndrome. I Hum Hypertens 22, 83-88

Iannuzzi A, Licenziati MR, Acampora C, Salvatore V, Auriemma L, Romano ML, Panico S, Rubba P, Trevisan M (2004) Increased carotid intima-media thickness and stiffness in obese children. Diabetes Care 27, 2506-2508

Inselma LS, Milanese A, Deurloo A (1993) Effect of obesity on pulmonary function in children. Pediatr Pulmonol 16, 130-137

Kiess W, Galler A, Reich A, Müller G, Kapellen T, Deutscher |, Raile K, Kratzsch I (2001) Clinical aspects of obesity in childhood and adolescence. Obes Rev 2, 29-36

Kromeyer-Hauschild K, Wabitsch M, Kunze D (2001) Perzentile für den Body-mass-Index für das Kindes-und Jugendalter unter He- ranziehung verschiedener deutscher Stichproben. Monatsschrift Kinderheilkunde 149, 807-808

Kurth BM, Schaffrath Rosario A (2007) Die Verbreitung von Übergewicht und Adipositas bei Kindern und Jugendlichen in Deutschland. Bundesgesundheitsbl 50, 736-743

Nafiu 00, Burke C, Cowan A, Tutuo N, Maclean S, Tremper KK (2010) Comparing peripheral venous access between obese and normal weight children. Paediatr Anaesth 20, 172-176

Nafiu 00, Green GE, Walton S, Morris M, Reddy S, Tremper KK (2009) Obesity and risk of peri-operative complications in children presenting for adenotonsillectomy. Int | Pediatr Otorhinolaryngol 73, 89-95

Nafiu 00, Maclean S, Blum I, Kheterpa S, Cowan A, Tremper KK (2010a) High BMI in children as a risk factor for intraoperative hypotension. Eur | Anaesthesiol 27, 1065-1068

Nafiu 00, Reynolds PI, Bamgbade OA, Tremper KK, Welch K, KasaVubu IZ (2007) Childhood body mass index and perioperative complications. Paediatr Anaesth 17, 426-430

Sagi R, Reif S, Neuman G, Webb M, Phillip M, Shalitin S (2007) Nonalcoholic fatty liver disease in overweight children and adolescents. Acta Paediatr 96, 1209-1213

Schwartz AE, Matteo RS, Ornstein E, Young WL, Myers KJ (1991) Pharmacokinetics of sufentanil in obese patients. Anesth Analg 73, 790-793

Setzer N, Saade E (2007) Childhood obesity and anesthetic morbidity. Paediatr Anaesth 17, 321-326

Shore SA (2010) Obesity, airway hyperresponsiveness, and inflammation. J Appl Phsiol 108, 735-743

Sorof J, Daniels S (2002) Obesity hypertension in children: a problem of epidemic proportions. Hypertension 40, 441-447

Srinivasan V, Nadkarni VM, Helfear MA; Cary SM, Berg RA (2010) Childhood obesity and survival after in-hospital pediatric cardiopulmonary resuscitation. Pediatrics 125 , e481-488

Strauß JM, Höhne C (2008) Adipositas - Risiko für die ambulante Anästhesie? Ambulant Operieren 2, 56-60

Tait AR, Voepel-Lewis T, Burke C, Kostrzewa A, Lewis I (2008) Incidence and risk factors for perioperative adverse respiratory events in children who are obese. Anesthesiology 108, 375-380

Toschke AM, Montgomery SM, Pfeiffer U, von Kries R (2003) Early intrauterine exposure to tobacco-inhaled products and obesity. Am | Epidemiol 158, 1068-1074

von Ungern-Sternberg BS, Saudan S, Petak F, Hantos Z, Habre W (2008) Desflurane but not sevoflurane impairs airway and respiratory tissue mechanics in children with susceptible airways. Anesthesiology 108, 216-224

Veyckemans F (2008) Child obesity and anaesthetic morbidity. Curr Opin Anaesthesiol 21, 308-312

Wang JH, Chung YS, Cho YW, Kim DY, Yi JS, Bae JS, Shim MJ (2010) Palatine tonsil size in obese, overweight, and normal-weight children with sleep-disordered breathing. Otolaryngol Head Neck Surg 142, 516-519

Weber E, Hiebl A, Storr U (2008) Prävalenz und Einflussfaktoren von Übergewicht und Adipositas bei Einschulungskindern. Dtsch Aerztebl 105, 883-889

Weiss R, Dziura J, Burgert TS, Tamborlane WV, Taksali SE, Yeckel CW, Allen K, Lopes M, Savoye M, Morrison I, Sherwin RS, Caprio S (2004) Obesity and the metabolic syndrome in children and adolescents. N Engl J Med; 350, 2362-2374 


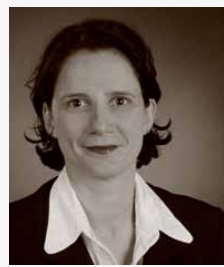

Prof. Dr. med. Claudia Philippi-Höhne

Prof. Dr. med. Claudia Philippi-Höhne arbeitet an der Klinik und Poliklinik für Anästhesiologie und Intensivtherapie des Universitätsklinikums Leipzig im Frauen-Kind-Zentrum als Oberärztin. Ihre Forschungsschwerpunkte liegen im Bereich der Kinderanästhesie und der angewandten Physiologie. 